\title{
Diagnosis With Manometry and Treatment With Repetitive Transcranial Magnetic Stimulation in Dysphagia
}

\author{
Won Ihl Rhee, MD, Sun Jae Won, MD, Sae Byulk Ko, MD \\ Department of Rehabilitation Medicine, Yeouido St. Mary's Hospital, \\ The Catholic University of Korea College of Medicine, Seoul, Korea
}

\begin{abstract}
Videofluoroscopic swallowing study (VFSS) used for the diagnosis of dysphagia has limitations in objectively assessing the contractility of the pharyngeal muscle or the degree of the upper esophageal sphincter relaxation. With a manometer, however, it is possible to objectively assess the pressure changes in the pharynx caused by pharyngeal muscle contraction during swallowing or upper esophageal sphincter relaxation, hence remedying the limitations of VFSS. The following case report describes a patient diagnosed with lateral medullar infarction presenting a 52-year-old male who had dysphagia. We suggested that the manometer could be used to assess the specific site of dysfunction in patients with dysphagia complementing the limitations of VFSS. We also found that repetitive transcranial magnetic stimulation was effective in treating patients refractory to traditional dysphagia rehabilitation.
\end{abstract}

Keywords Manometry, Dysphagia, Transcranial magnetic stimulation

\section{INTRODUCTION}

Dysphagia is common after stroke, occurring in $23 \%-$ $50 \%$ of patients [1]. Dysphagia may result from a lesion between the corticobulbar tract spanning the cerebral cortex to the brainstem. In particular, the central pattern generator involved in the swallowing reflex is located

Received February 6, 2013; Accepted June 11, 2013

Corresponding author: Sae Byuk Ko

Department of Rehabilitation Medicine, Yeouido St. Mary's Hospital, The Catholic University of Korea College of Medicine, 10 63(yuksam)-ro, Yeongdeungpo-gu, Seoul 150-713, Korea

Tel: +82-2-3779-1257, Fax: +82-2-786-4502, E-mail: sae_byuk@hotmail.com

(c) This is an open-access article distributed under the terms of the Creative Commons Attribution Non-Commercial License (http://creativecommons. org/licenses/by-nc/3.0) which permits unrestricted noncommercial use, distribution, and reproduction in any medium, provided the original work is properly cited.

Copyright $\odot 2013$ by Korean Academy of Rehabilitation Medicine at the medulla. It receives neurotransmissions from the cerebral cortex via the bilateral corticobulbar tract, and so is involved in the regulation of the pharyngeal and esophageal muscles and peristalsis via the nucleus ambiguous and dorsal nucleus of the vagus nerve. Serious dysphagia that can occur as a small lesion of the medulla can directly affect the central pattern generators for swallowing $[2,3]$. The opening of the upper esophageal sphincter is associated with the relaxation of the cricopharyngeal muscle and laryngeal superior-anterior movement, and the laryngeal movement is caused by the contraction of the suprahyoid muscle and thyrohyoid muscle. The coordinated movement of the pharynx, larynx, and esophagus is important. These processes are regulated by the aforementioned central pattern generators for swallowing [4]. Lower pharyngeal retention observed by videofluoroscopic swallowing study 
(VFSS) may occur due to the decreased contractility of the pharyngeal muscle or the dysfunctional relaxation of the upper esophageal sphincter, and the impairment of the coordinated movement of pharyngeal contraction between the relaxation of the upper esophageal sphincter [5]. With VFSS alone, however, it is difficult to accurately discriminate which causes the lower pharyngeal retention. Manometry, which objectively measures pressure changes in the pharynx caused by the pharyngeal muscle contraction and pressure changes caused by the relaxation of the upper esophageal sphincter during swallowing, can complement the disadvantages of VFSS. The result can be a detailed diagnosis, leading to a more appropriate treatment. Only a few studies have applied repetitive transcranial magnetic stimulation (rTMS) to patients with post-stroke dysphagia that occurred at the cerebral hemisphere and brainstem. These studies reported that dysphagia was improved. Accordingly, based on the results of the previous studies, authors present a case highlighting the usefulness of manometry and the effect of rTMS treatment following VFSS in a patient with post-lateral medullary infarction dysphagia who was refractory to conventional dysphagia rehabilitation.

\section{CASE REPORT}

A 52-year-old male patient visited the emergency department in our hospital due to sudden dizziness and dysphagia. The patient was diagnosed with lateral medullary infarction using brain magnetic resonance imaging. Three days after onset, he was referred to the department of rehabilitation medicine for the rehabilitative treatment of dysphagia. The patient had right blepharoptosis and no muscle weakness except for ataxia of the right lower extremity. He was being fed by a gastric feeding tube. Water swallowing test demonstrated a typical wet voice and laryngopharyngeal retention despite the small amount of food intake. VFSS was conducted to identify the causative factors of dysphagia 4 days after the onset. The functional dysphagia scale score was 60 points, with no particular abnormality in the anterosuperior elevation of the oral cavity and larynx, atresia of the epiglottis, definite contraction of the upper pharynx, and retention of almost all foods in the pyriformis sinus and vallecular fossa after swallowing, with no passage past the upper esophageal sphincter (Table 1). During the test, compensation techniques, such as Mendelsohn maneuver, head turn, and head lean, were also conducted, but no particular difference was observed. The patient underwent rehabilitative treatments that included electrical stimulation for dysphagia, Shaker exercise, and training concerning a compensation technique including the Mendelsohn maneuver. An assessment was conducted using esophageal manometry 32 days after onset to more accurately identify the cause of dysphagia. By setting the upper esophageal sphincter as a reference point, the pressure changes in the pharynx above the upper esophageal sphincter, upper esophageal sphincter, and esophageal body were measured. Normal pressure change in the upper pharynx, decreased contraction of the lower pharynx, definitely decreased pressure upon the relaxation of the upper esophageal sphincter, retention in the upper esophageal sphincter due to the impairment of the coordination with the contraction of the lower pharynx, and no pressure changes caused by esophageal peristalsis were evident (Fig. 1). About four weeks later (33 days after onset), VFSS was conducted again. The functional dysphagia scale score was again 60 points (Table 1). As the patient was refractory to the one-month dysphagia rehabilitative treatment including electrical treatment for dysphagia, Shaker exercise, and the training of compensation technique, another treatment was applied.

As several previous studies reported that dysphagia was improved by increasing the neurotransmission and plasticity of the corticobulbar tract via rTMS of the cerebral cortex associated with swallowing [6,7], rTMS was used. The International 10-20 system and a bandana with lattice positioned at intervals of $1 \mathrm{~cm}$ were used to determine the stimulation site. The surface electrode was used to determine the site with the lowest motor threshold of the pharyngeal-laryngeal muscles, which was identified as an area $2 \mathrm{~cm}$ anterior and $5 \mathrm{~cm}$ lateral from the vertex of the head. rTMS was conducted with a frequency of 5 $\mathrm{Hz}$ and intensity of $120 \%$ of the motor threshold of bilateral hemisphere, and 10 cycles of 10 seconds stimulation and 50 seconds relaxation for 10 days (five times/week for 2 weeks) followed by VFSS and manometry to assess the result of the 2-week TMS treatment. The functional dysphagia scale was improved to 20 points. Compared to the result of the preceding VFSS, which showed that most foods were retained in the lower pharynx because of the inability to passing by the upper esophageal sphincter, 
Table 1. Functional dysphagia scale

\begin{tabular}{|c|c|c|c|c|}
\hline & Score & $\begin{array}{c}4 \text { days } \\
\text { after onset }\end{array}$ & $\begin{array}{c}33 \text { days } \\
\text { after onset }\end{array}$ & $\begin{array}{l}47 \text { days } \\
\text { after onset }\end{array}$ \\
\hline \multicolumn{5}{|l|}{ Lip closure } \\
\hline Intact & 0 & $\mathrm{O}$ & $\mathrm{O}$ & $\mathrm{O}$ \\
\hline Inadequate & 5 & & & \\
\hline None & 15 & & & \\
\hline \multicolumn{5}{|c|}{ Bolus formation } \\
\hline Intact & 0 & $\mathrm{O}$ & $\mathrm{O}$ & $\mathrm{O}$ \\
\hline Inadequate & 3 & & & \\
\hline None & 6 & & & \\
\hline \multicolumn{5}{|c|}{ Residue in the oral cavity (\%) } \\
\hline None & 0 & $\mathrm{O}$ & $\mathrm{O}$ & $\mathrm{O}$ \\
\hline$\leq 10$ & 2 & & & \\
\hline $10-50$ & 4 & & & \\
\hline$>50$ & 6 & & & \\
\hline \multicolumn{5}{|c|}{ Oral transit time (sec) } \\
\hline$\leq 1.5$ & 0 & $\mathrm{O}$ & $\mathrm{O}$ & $\mathrm{O}$ \\
\hline$>1.5$ & 6 & & & \\
\hline \multicolumn{5}{|c|}{ Triggering of pharyngeal swallowing } \\
\hline Normal & 0 & & & $\mathrm{O}$ \\
\hline Delayed & 10 & $\mathrm{O}$ & $\mathrm{O}$ & \\
\hline \multicolumn{5}{|c|}{ Laryngeal elevation and epiglottic closure } \\
\hline Normal & 0 & & & \\
\hline Reduced & 12 & $\mathrm{O}$ & $\mathrm{O}$ & $\mathrm{O}$ \\
\hline \multicolumn{5}{|c|}{ Nasal penetration (\%) } \\
\hline None & 0 & $\mathrm{O}$ & $\mathrm{O}$ & $\mathrm{O}$ \\
\hline$\leq 10$ & 4 & & & \\
\hline $10-50$ & 8 & & & \\
\hline$\geq 50$ & 12 & & & \\
\hline \multicolumn{5}{|c|}{ Residue in the valleculae } \\
\hline None & 0 & & & \\
\hline$\leq 10$ & 4 & & & $\mathrm{O}$ \\
\hline $10-50$ & 8 & & & \\
\hline$\geq 50$ & 12 & $\mathrm{O}$ & $\mathrm{O}$ & \\
\hline \multicolumn{5}{|c|}{ Residue in the pyriform sinuses } \\
\hline None & 0 & & & \\
\hline$\leq 10$ & 4 & & & $\mathrm{O}$ \\
\hline $10-50$ & 8 & & & \\
\hline$\geq 50$ & 12 & $\mathrm{O}$ & $\mathrm{O}$ & \\
\hline \multicolumn{5}{|c|}{ Coating of pharyngeal wall after swallowing } \\
\hline No & 0 & & & $\mathrm{O}$ \\
\hline Yes & 10 & $\mathrm{O}$ & $\mathrm{O}$ & \\
\hline \multicolumn{5}{|c|}{ Pharyngeal transit time (sec) } \\
\hline$\leq 1.0$ & 0 & & & $\mathrm{O}$ \\
\hline$>1.0$ & 4 & $\mathrm{O}$ & $\mathrm{O}$ & \\
\hline Total score & 100 & 60 & 60 & 20 \\
\hline
\end{tabular}



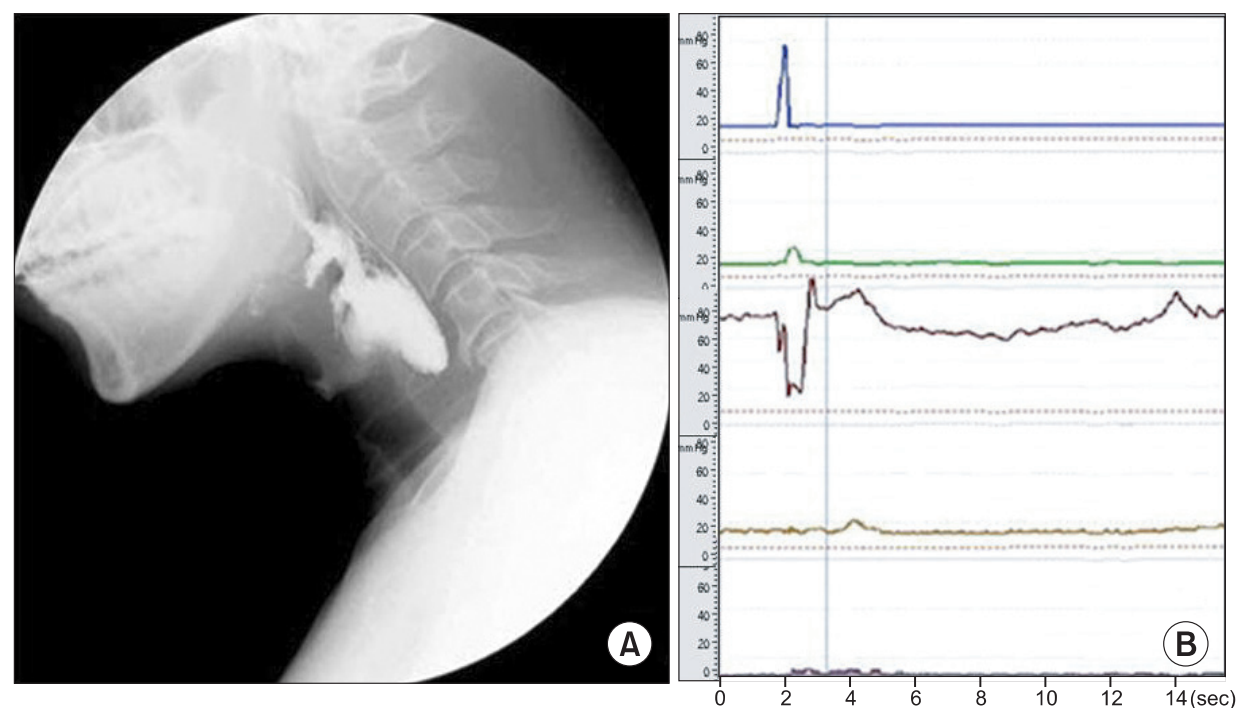

Fig. 1. Videofluoroscopic swallowing study finding (A) and manometry finding (B) before repetitive transcranial magnetic stimulation. The pressure changes in the pharynx and oesophagus were recorded using a 5-lumen perfused catheter system. The plots are: $10 \mathrm{~cm}$ upper of the UES (blue line), $5 \mathrm{~cm}$ upper of the UES (green line), UES (brown line), $5 \mathrm{~cm}$ lower of the UES (yellow line), $10 \mathrm{~cm}$ lower of the UES (purple line). The pressure increase was reduced at the site $5 \mathrm{~cm}$ upper of the UES (green line). The decreased pressure due to UES relaxation was clear (brown line), but the retained foods not passing by the UES due to the reduced duration was observed. In addition, no pressure change caused by peristalsis was observed due to the retained foods did not pass by the esophageal body. UES, upper esophageal sphincter.
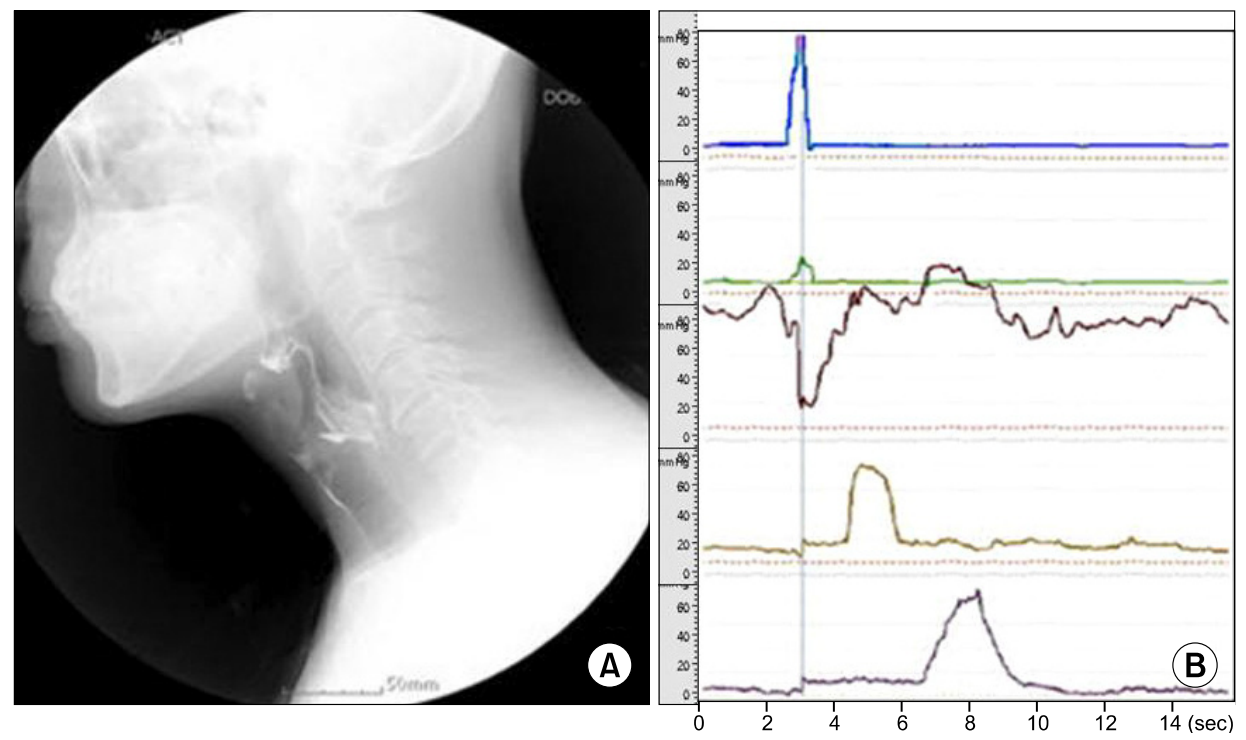

Fig. 2. Videofluoroscopic swallowing study finding (A) and manometry finding (B) after the 2-week repetitive transcranial magnetic stimulation treatment. Compared to the status observed 2 weeks prior, no significant increase in the pressure change at the site $5 \mathrm{~cm}$ upper of the UES was observed. However, foods passed by the UES due to the extended duration of UES relaxation, and peristalsis were also observed in the esophageal body. UES, upper esophageal sphincter. 
at the follow-up most foods were able to passed by the upper esophageal sphincter. A small quantity of food was retained in the pyriformis sinus and vallecular fossa sinus after swallowing, and the laryngeal delay time was significantly shortened (Table 1, Fig. 2). Meanwhile, compared to previous manometry results, the follow-up revealed a prolonged relaxation time of the upper esophageal sphincter of 2 seconds, which was more than twice the time of the previous results. This would be sufficient enough to enable foods to pass by the upper esophageal sphincter after the contraction of the lower pharynx. The pressure change was observed in the esophageal phase due to peristalsis (Fig. 2).

After 2 weeks of rTMS, the patient began to consume small amounts of food in accordance with the schedule set by the authors. Regular chest X-ray and blood test revealed no complications, such as aspiration pneumonia, were observed. When the patient was able to consume 1,650 calories per day, he was discharged. He has been followed-up in our out-patient clinic and has been taking a regular diet without any complication for 2 years.

\section{DISCUSSION}

VFSS sometimes shows that the large amounts of food are retained in the lower pharynx. If even a small amount of foods cannot pass by the upper esophageal sphincter, as shown in the patient of this case study, the symptom is generally diagnosed as the impairment of upper esophageal sphincter relaxation. Thus, relaxation using a Foley catheter or injection of botulinum toxin is conducted to improve dysphagia. However, as shown in the case, retention of a large amount of food in the lower esophagus could be also caused by the impairment of the coordinated contractility of the lower pharynx or the reduced contraction of the lower pharynx, in addition to the impairment of relaxation of the upper esophageal sphincter. Thus, it is difficult to identify the exact cause of dysphagia using VFSS alone, which may lead to inappropriate treatment if VFSS is conducted alone in patients with dysphagia. Considering the limitation of VFSS and that other methods, such as manometry, are recommended for use along with VFSS for a more exact assessment, we presently used both approaches. VFSS can detect the abnormal movement of pharyngeal components during swallowing, such as the qualitative reduction of structure motil- ity related to swallowing, including reduced laryngeal superior-anterior movement. Meanwhile, manometry can measure pressure vector affecting the bolus, and assess upper esophageal sphincter relaxation and the contractility of the lower pharynx. In addition, it can assess the coordination by providing information on space-time pattern between the upper esophageal sphincter and the lower pharynx.

Non-invasive brain stimulation methods, such as rTMS or transcranial direct current stimulation, have recently drawn attention in the treatment of post-stroke complications. Fukuoka et al. [8] reported that the outcome of lateral medullar infarction with more rostral lesions tended to be poor. Since the lesion of the patient in our case was located on the rostrolateral medulla, prognosis was expected to be poor. Therefore, rTMS was applied as another treatment option. The hyperreflexic and incoordinated nature of upper esophageal sphincter would be related with the loss of inhibitory corticobulbar fiber. As shown in this case, lesion of lateral medullary infarction is unilateral, but the central pattern generators for swallowing receive stimulation via the bilateral corticobulbar tract. Thus, based on the hypothesis that the repetitive stimulation of the bilateral cerebral cortex via rTMS increases the excitability of the corticobulbar tract spanning from the cerebral cortex to the medulla, we suggest that an increase in the activity in the motor cortex by cortical stimulation would increase its inhibitory influence on the corticobulbar tract and, thus, reduce the hyperactivity of upper esophageal sphincter muscle [9]. Accordingly, rTMS was used. Significantly improved outcomes resulted compared to the pre-treatment status.

This case study has limitations. Dysphagia of the patient was relieved within 2 months after onset, which was an acute phase. Therefore, the possibility of spontaneous recovery cannot be excluded. On the other hand, conventional dysphagia rehabilitation had no effect, with improvement evident only after the 2-week rTMS. rTMS may have been an effective trigger for a spontaneous recovery. Further studies are needed to verify this speculation. In our setting, manometry had the disadvantage of low resolution due to a $5-\mathrm{cm}$ distance between the sensors, which led to an inevitable gap between the recording sites, thereby making it difficult to more precisely observe the contraction and relaxation between the gap. For more accurate measurement, high resolution manom- 
etry, which measures pressure changes at an interval of 1

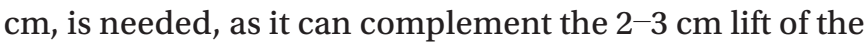
upper esophageal sphincter by laryngeal elevation during swallowing. In addition, a more accurate correlation of the results of VFSS and manometry can be achieved if the manofluorography that can simultaneously conduct both tests, compared to the separate conducts of VFSS and manometry shown in this case study.

\section{CONFLICT OF INTEREST}

No potential conflict of interest relevant to this article was reported.

\section{REFERENCES}

1. Singh S, Hamdy S. Dysphagia in stroke patients. Postgrad Med J 2006;82:383-91.

2. Sessle BJ, Henry JL. Neural mechanisms of swallowing: neurophysiological and neurochemical studies on brain stem neurons in the solitary tract region. Dysphagia 1989;4:61-75.

3. Higo R, Tayama N, Watanabe T. Manometric abnormality in dysphagic patients after medullary cerebro- vascular accidents. ORL J Otorhinolaryngol Relat Spec 2002;64:368-72.

4. Jean A. Brainstem organization of the swallowing network. Brain Behav Evol 1984;25:109-16.

5. Hila A, Castell JA, Castell DO. Pharyngeal and upper esophageal sphincter manometry in the evaluation of dysphagia. J Clin Gastroenterol 2001;33:355-61.

6. Khedr EM, Abo-Elfetoh N. Therapeutic role of rTMS on recovery of dysphagia in patients with lateral medullary syndrome and brainstem infarction. J Neurol Neurosurg Psychiatry 2010;81:495-9.

7. Jefferson S, Mistry S, Michou E, Singh S, Rothwell JC, Hamdy S. Reversal of a virtual lesion in human pharyngeal motor cortex by high frequency contralesional brain stimulation. Gastroenterology 2009;137:841-9.

8. Fukuoka T, Takeda H, Dembo T, Nagoya H, Kato $\mathrm{Y}$, Deguchi I, et al. Clinical review of 37 patients with medullary infarction. J Stroke Cerebrovasc Dis 2012;21:594-9.

9. Ertekin C, Turman B, Tarlaci S, Celik M, Aydogdu I, Secil Y, et al. Cricopharyngeal sphincter muscle responses to transcranial magnetic stimulation in normal subjects and in patients with dysphagia. Clin Neurophysiol 2001;112:86-94. 